\title{
Efficacy of G32 in Treating Pregnancy Gingivitis A Randomised Controlled Trial
}

\author{
Research Article
}

\begin{abstract}
Hathiwala $\mathbf{S}^{1^{*}}$, Acharya $S^{2}$, Bhat $\mathbf{P V}^{3}$, Patil $S^{1}$
1. Post Graduate, 2. Professor and Head of Department, Dept. of Public Health Dentistry, Manipal College of Dental Sciences, Manipal, Karnataka, 3. Professor and Head of Department, Dept. of Obstetrics and Gynaecology, Maleka Manipal Medical College, Manipal, Karnataka, India
\end{abstract}

\begin{abstract}
Background: Pregnant females are susceptible to oral conditions like gingivitis and pregnancy tumour, which affect both the mother and the developing foetus. Chlorhexidine, conventionally used to treat these conditions, causes side effects on long term use. Ayurvedic medications like $\mathrm{G} 32$ have been used for treating gingivitis.

Objective: To compare efficacy of G32 with Chlorhexidine in treating pregnancy gingivitis after topical application.

Materials and Methods: This was a double blind, randomised controlled trial conducted in a sample of 37 pregnant females, in second trimester, with gingivitis. They were randomly divided into two groups and were allotted Chlorhexidine gel ( $\mathrm{n}=18)$ or G32 gumpaint $(n=19)$ for local application for one month. Plaque index (Silness and Löe, 1964) and Gingival index (Löe and Silness, 1963) were recorded at baseline and follow-up by a calibrated examiner. Statistical analysis was performed using paired t-test for intragroup comparison of mean plaque and gingival scores, and independent t-test for intergroup comparison of mean percentage reduction in plaque and gingival scores.

Results: Both test and control groups showed a significant reduction in plaque and gingivitis. The percentage reductions in plaque and gingivitis were similar among the two groups.

Conclusion: G32 is effective in treating pregnancy gingivitis and can be economical alternative to Chlorhexidine, with lesser side effects.

Keywords: Ayurvedic, Pregnancy, Gingivitis, Chlorhexidine, G32, Trial

\section{Introduction:}

Pregnancy has far-reaching systemic effects extending beyond the reproductive organs. These effects are the

*Corresponding Author:

\section{Hathiwala $S$}

Post Graduate (MDS),

Dept. of Public Health Dentistry,

Manipal College of Dental Sciences,

Manipal

Karnataka, India

E-mail: siddhi.hathiwala@gmail.com

Ph.No: +91 9741544482

end results of complex hormonal, immunologic, dietary, and behavioural changes occurring during pregnancy (1). These can include changes in the cardiovascular, respiratory and gastrointestinal systems, as well as changes in the oral cavity and increased susceptibility to oral infection (2). The oral effects are primarily seen as gingival manifestations; mainly causing pregnancyassociated gingivitis and pyogenic granuloma or pregnancy tumour. So maintaining a good oral health is important during pregnancy.
\end{abstract}


The occurrence of pregnancy gingivitis has been acknowledged for a substantial period of time. The term was first described in 1877 (3), although descriptions of the condition had been given even earlier in 1840 (4). It is extremely common and affects $30-75 \%$ of all pregnant women $(5,6)$.

Gingival inflammatory changes in pregnancy usually begin during the second month and increase in severity through the eighth month, after which an abrupt decrease occurs in the symptoms $(7,8)$. The greatest involvement appears to be in gingivae of the anterior teeth, with interproximal sites most commonly affected (9). The interproximal papillae become red, oedematous and tender to palpation, and they bleed easily if subjected to trauma (2). Various studies have confirmed that the prevalence and severity of gingival inflammation is significantly higher in pregnant women compared to postpartum, and appears unrelated to the amount of plaque present. Also pre-existing gingivitis or periodontitis in pregnant women has been noted to worsen dramatically (9).

Even a healthy pregnancy causes major changes in maternal anatomy, physiology and metabolism. These patients have a heightened awareness of and sensitivity to taste, smell and environmental temperature. Unpleasant tastes and odours can cause severe nausea or even gagging and vomiting (2). Although these adaptations of maternal body are normal, they do necessitate consideration and adjustments in treatment by any dentist, who is providing oral health care and prescribing medications for the patient.

Routine general dentistry should be done in the second trimester of pregnancy only, due to organogenesis occurring during the first trimester and increased uterine size in the third trimester, making sitting in the dental chair uncomfortable. Also nausea generally ceases by the end of the first trimester. Any treatment should be directed toward controlling disease, maintaining a healthy oral environment and preventing potential problems that could occur later in pregnancy or during the postpartum period.

Nevertheless, a treatment for gingivitis is definitely needed, as the lack of care of gums and teeth affects both the mother and the foetus. Severe gingival disease during pregnancy and the periodontal disease, which progresses from untreated gingivitis, can lead to premature delivery and low birth weight of the baby $(10,11)$. Thorough oral hygiene measures, including tooth brushing and flossing, are recommended to prevent occurrence of these diseases. Patients with severe gingivitis may require professional cleaning, along with mouth rinses like Chlorhexidine. More severe cases, if do not regress, need surgical therapy (12) which is not advisable during pregnancy. So the non-surgical treatment is the best approach.

Chlorhexidine is a known therapeutic agent used for treating gingivitis in general and also in pregnancy gingivitis. It is a dicationic bisguanide with pronounced antiseptic, antibacterial, antifungal and antiplaque properties. It is available as oral rinse $(0.2 \%$ and $0.12 \%)$ and gel $(1 \%)$. Many studies have shown that the long term use of Chlorhexidine may cause side effects ranging from as mild as staining of oral surfaces, such as tooth surfaces, restorations, and the dorsum of the tongue and a transient alteration in taste perception, to as severe as hypersensitivity, generalized allergic reactions and oro-pharyngeal cancer (13). High content of alcohol (12\%) in this product has been a point of concern for use in pregnancy and so it has been placed under category ' $\mathrm{B}$ ' for usage in pregnant females (14).

Under such circumstances, it has become vital to explore safe alternatives to Chlorhexidine. Many ayurvedic 
formulations have been tested and have been proved to be effective in treating gingival diseases, with fewer deleterious effects. Many formulations like Salvadora persica (Miswak), Triphala, Propolis, Azadirachta indica (Neem) have been widely used (15-18).

G32, used in this study, is one such ayurvedic preparation. It is available for local application, in an easily crushable tablet form and as gum paint. The main ingredients in this ayurvedic preparation include Bakul, Chok, Katha, Laving, Fatakdi, etc. (Appendix A). It has antiinflammatory, antiseptic, antibacterial, astringent, anodyne, styptic and healing actions. Various studies have shown it to be effective in treating gingivitis (19-21) and pregnancy-induced gingivitis (22). These studies have shown that it has minimal side effects and has good compliance of the patients. However, no studies have been conducted till this date that compare G32 with Chlorhexidine, the existing 'gold standard' for gingivitis (23). So this study was conducted with an aim to compare the effectiveness of ayurvedic formulation, G32 with Chlorhexidine in treating pregnancy gingivitis.

$\begin{array}{llr}\text { Constituents of G32 (19-22) } & \\ \text { Bakul (Mimosops elangi) } & - & 80 \mathrm{mg} . \\ \text { Chok (Calcium Carbonate) } & - & 75 \mathrm{mg} . \\ \text { Katho (Acacia catechu) } & - & 40 \mathrm{mg} . \\ \text { Laving (Myrtus } & & \\ \text { caryophyllus) } & & \\ \text { Chikani Sopari (Areca } & & \mathrm{mg} . \\ \text { catechu) } & - & 20 \mathrm{mg} . \\ \text { Fatakadi (Alumen) } & - & 20 \mathrm{mg} . \\ \text { Mayafal (Quercus infectoria) } & - & 20 \mathrm{mg} . \\ \text { Elaichi (Elettaria } & - & 10 \mathrm{mg} . \\ \text { cardamomum) } & \\ \begin{array}{l}\text { Sonageru (Silicate of } \\ \text { Alumina and Iron Oxide) }\end{array} & - & 10 \mathrm{mg} . \\ \text { Jiru (Carum carvi) } & \\ \begin{array}{l}\text { Majith (Rubia cordifolia) } \\ \text { Pashanbed (Saxifrua }\end{array} & - & 10 \mathrm{mg} . \\ \text { ligulata) } & - & 10 \mathrm{mg} . \\ \text { Vavding (Embelia ribes) } & - & 10 \mathrm{mg} .\end{array}$

\begin{tabular}{|c|c|}
\hline & \\
\hline Samudrafin (Os sapiae) & \\
\hline Vajradanti (Barleria prioitis) & \\
\hline Taj (Cinnamimum cassia) & \\
\hline Mari (Piper nigrum) & \\
\hline $\begin{array}{l}\text { Sajikhar (Sodium carbonate } \\
\text { impure) }\end{array}$ & \\
\hline Kulinjan (Alpinia chinensis) & \\
\hline Pipar (Piper longum) & \\
\hline $\begin{array}{l}\text { Kapur (Camphora } \\
\text { officinarum) }\end{array}$ & \\
\hline Kuth (Uncaria gambier) & \\
\hline
\end{tabular}

Objectives:

- To evaluate the effectiveness of G32 gum-paint in reducing dental plaque and gingivitis in pregnant females.

- To compare its effectiveness with Chlorhexidine gel.

\section{Materials and methods:}

This was a parallel design, randomised controlled trial, conducted on pregnant females with gingivitis, in their second trimester. The examinations were performed in the Ante-natal Gynaecological clinics of Dr. T.M.A. Pai Hospital, Udupi (Karnataka, India). The study was approved by the Kasturba Hospital Institutional Ethics Committee, Manipal (IEC 82/2011). An informed consent was taken from all the study participants before their inclusion.

\section{Sample selection -}

- The pregnant females, aged 20-35 years, who were in their second trimester and had signs of moderate to severe gingivitis, with or without plaque or debris, were included in the study.

- Those who had medical complications related to pregnancy, or any medical disorders or chronic/acute infections other than dental infection, requiring any kind of intervention, or reported a recent history of antibiotic 
administration for any reason, were excluded from the sample.

The final sample comprised of 37 pregnant females who were randomly divided into two groups. 19 females in the test group were given G32 Alarsin gumpaint (ALARSIN, Mumbai), while 18 others formed the control group and were given Chlorhexidine gel (Hexigel, ICPA, Mumbai). Both the groups were instructed to apply the medicament and massage it over gums twice a day, along with their existing oral hygiene practices. The effects of the medicaments were assessed after one month of usage.

\section{Examination}

This trial was conducted from March, 2011 to June, 2011. All the pregnant females attending regular antenatal check-ups in the Gynaecology Dept. were examined for their oral health status by the principal investigator, while general medical examination was performed by other investigator. The females with moderate to severe gingivitis (gingival scores $\geq 1$ ) were included in the study after taking consent.

The participants' information relating their demographics (age, education, income and address), months of pregnancy, personal oral habits, oral hygiene practices were recorded by one of the investigators, after interviewing them. Self-perceived oral health status was also recorded on a 5-point Likert scale, ranging from 'excellent' to 'poor'.

This was followed by clinical oral examination which was performed by the principal investigator. The Gingival and Plaque scores were assessed using the Löe and Silness Index (1963) (24) and Silness and Löe Index (1964) (25), respectively.
These indices were calculated on selected maxillary and mandibular teeth for all the patients, using a mouth mirror and dental probe. The Gingival scores ranged from 0 3 depending on the severity of inflammation in gingival margin. Similarly, the Plaque scores ranged from 0-3 depending upon the thickness of plaque at gingival area of the tooth. Only one trained examiner did all the examinations to prevent the inter-examiner bias. Intra-examiner reliability was assessed by the kappa coefficient. The kappa values for Gingival and Plaque scores were 0.81 and 0.78 respectively.

Followed by this, the participants were randomly allotted one of the two medicaments in wrapped bottles, for one month usage. These bottles were sequentially numbered by an investigator who was not a part of the clinical examination. The subjects were instructed to thoroughly massage the medicament over gums and surrounding areas, with the help of finger-tip and were advised to keep it for minimum five minutes, and then rinse with fresh water, without swallowing it. This procedure was to be repeated twice daily for the first 15 days, in morning and at bed time.

\section{Interim Review}

After the first 15 days, a reminder call was made by the investigator to enquire about the compliance to the instructions. The patients were asked if they experienced any change in the condition of their gums. They were instructed to continue using the medicament once daily (in morning) for the next 15 days. 


\section{Follow-up}

All the females were examined at the end of one month for all the parameters evaluated at the baseline. The side effects, if any, were also recorded at this visit. The females were instructed for better oral hygiene methods and importance of oral health in pregnancy was reinforced. They were also advised for a dental visit soon after their delivery.

\section{Data analysis}

All the data were analysed using the SPSS version 16.0. The intragroup changes in pre-test and post-test plaque and gingival scores were evaluated using a Paired t-test. Post-test plaque and gingival scores between the test and control groups were compared using Independent t-test. A p-value $\leq 0.05$ was considered to be statistically significant.

\section{Results}

A total of 400 pregnant females in their second trimester were examined, and 69 females that fulfilled the inclusion criteria were selected. From these, 37 females consented to participate in the study. The most common reason for not participating in the study was apprehension on part of the mothers about the possible risk to their foetuses. The females, who consented, were randomly divided into Chlorhexidine $(\mathrm{n}=18)$ and G32 $(n=19)$ groups.

Out of these 37 pregnant females who were examined at the baseline, about $80 \%$ (29 females) perceived their oral health status as 'fair'. None of them perceived their oral status as 'excellent'.

Three females were lost to followup of 4 weeks. Two females prematurely withdrew from the study, because of nausea; one each from the test and the control groups. One female from the test group failed to return for the post-test examination. Hence, the final sample of 34 females was analysed, with 17 patients in each group.

The mean age of the females in Chlorhexidine and G32 groups was 28.3 ( \pm $2.1)$ and $26.5( \pm 3.6)$ years respectively, with a range of 20-33 years. The mean duration of pregnancy was $4.52( \pm 0.8)$ and $4.32( \pm 0.9)$ months respectively, with a range of 3-6 months. The mean baseline plaque scores were $1.93( \pm 0.5)$ and 1.64 $( \pm 0.4)$ for Chlorhexidine and G32 groups respectively. Similarly, the mean baseline gingival scores were $1.94( \pm 0.5)$ and 1.76 $\left(\begin{array}{ll} \pm & 0.3\end{array}\right)$ respectively. There was no statistically significant difference between the test and control groups for mean age ( $p$ $=0.08)$, duration of pregnancy $(\mathrm{p}=0.49)$, and plaque $(\mathrm{p}=0.06)$ and gingival $(\mathrm{p}=$ 0.22 ) scores. (Table 1)

Table 1 - Baseline characteristics of participants of Chlorhexidine and G32 groups

\begin{tabular}{|c|c|c|c|c|c|}
\hline Treatment & $\begin{array}{c}\text { No. of } \\
\text { Patients }\end{array}$ & $\begin{array}{c}\text { Mean Age } \\
\text { (years) }\end{array}$ & $\begin{array}{c}\text { Mean duration } \\
\text { of pregnancy } \\
\text { (months) }\end{array}$ & $\begin{array}{c}\text { Mean Plaque } \\
\text { Scores }( \pm \text { SD) }\end{array}$ & $\begin{array}{c}\text { Mean Gingival } \\
\text { Scores }( \pm \text { SD) }\end{array}$ \\
\hline Chlorhexidine & 17 & 28.37 & 4.52 & $1.93 \pm 0.52$ & $1.94 \pm 0.49$ \\
\hline G32 & 17 & 26.59 & 4.32 & $1.64 \pm 0.33$ & $1.76 \pm 0.32$ \\
\hline
\end{tabular}

The plaque scores reduced from $1.93( \pm 0.5)$ to $0.79( \pm 0.4)$ in the Chlorhexidine group, while in G32 group, a reduction from $1.64( \pm 0.4)$ to $0.87( \pm 0.4)$ was seen in the 
plaque scores. The paired t-test showed that reduction in the plaque scores in both the study groups was statistically significant $(\mathrm{p}=0.001)$. (Table 2)

Table 2 - Comparison of pre- and post-test plaque scores in both the study groups

\begin{tabular}{|c|c|c|c|c|c|}
\hline Treatment & $\begin{array}{c}\text { Pre-test plaque } \\
( \pm \mathrm{SD})\end{array}$ & $\begin{array}{c}\text { Post-test plaque } \\
( \pm \mathrm{SD})\end{array}$ & $\begin{array}{c}\text { Mean } \\
\text { Difference } \\
( \pm \mathrm{SD})\end{array}$ & $\begin{array}{c}\text { Percentage } \\
\text { reduction }\end{array}$ & P-value \\
\hline Chlorhexidine & $1.93 \pm 0.52$ & $0.79 \pm 0.39$ & $1.14 \pm 0.48$ & $59 \%$ & $0.001^{*}$ \\
\hline G32 & $1.64 \pm 0.33$ & $0.87 \pm 0.41$ & $0.77 \pm 0.38$ & $52.9 \%$ & $0.001 *$ \\
\hline
\end{tabular}

* - P-value $<0.05$ considered significant

Paired t-test - Comparing intragroup pre- and post- plaque scores

The gingival scores reduced from $1.94( \pm 0.5)$ to $0.75( \pm 0.3)$ in the Chlorhexidine group, while in G32 group, these reduced from $1.76( \pm 0.3)$ to $0.72( \pm 0.4)$. This reduction in both the study groups was found to be statistically significant $(\mathrm{p} \leq 0.001)$. (Table 3)

Table 3 - Comparison of pre- and post-test gingival scores in both the study groups

\begin{tabular}{|c|c|c|c|c|c|}
\hline Treatment & $\begin{array}{c}\text { Pre-test } \\
\text { gingivitis } \\
( \pm \text { SD })\end{array}$ & $\begin{array}{c}\text { Post-test } \\
\text { gingivitis } \\
( \pm \mathrm{SD})\end{array}$ & $\begin{array}{c}\text { Mean } \\
\text { Difference } \\
( \pm \mathrm{SD})\end{array}$ & $\begin{array}{c}\text { Percentage } \\
\text { reduction }\end{array}$ & P-value \\
\hline Chlorhexidine & $1.94 \pm 0.49$ & $0.75 \pm 0.32$ & $1.18 \pm 0.37$ & $61 \%$ & $<0.001^{*}$ \\
\hline G32 & $1.76 \pm 0.32$ & $0.72 \pm 0.38$ & $1.04 \pm 0.44$ & $58.7 \%$ & $0.001^{*}$ \\
\hline
\end{tabular}

* - P-value $<0.05$ considered significant

Paired t-test - Comparing intragroup pre- and post- gingival scores

The plaque scores showed a mean percentage reduction of $59 \%$ in Chlorhexidine group and 53\% in G32 group. This difference was not found to be statistically significant following the independent t-test $(\mathrm{p}=0.48)$. The gingival scores showed a mean percentage reduction of $61 \%$ in Chlorhexidine group and $58 \%$ in G32 group. The independent t-test showed that this difference was not statistically significant $(p=0.64)$. (Table 4$)$

Table 4 - Comparison of the percentage reduction of plaque and gingival scores between Chlorhexidine and G32 groups -

\begin{tabular}{|c|c|c|c|}
\hline Characteristics & $\begin{array}{c}\text { Percentage reduction } \\
\text { Chlorhexidine }\end{array}$ & $\begin{array}{c}\text { Percentage } \\
\text { reduction G32 }\end{array}$ & P-value \\
\hline Plaque Scores & $58 \%$ & $53 \%$ & 0.484 \\
\hline Gingival Scores & $61 \%$ & $58 \%$ & 0.64 \\
\hline
\end{tabular}

P-value $<0.05$ considered significant

Independent t-test - Comparing intergroup changes in scores

\section{Discussion:}

This study was a parallel designed, double blind, randomised controlled clinical trial conducted on pregnant females with gingivitis, in their second trimester. It aimed to assess the effectiveness of $\mathrm{G} 32$ as compared to 
Chlorhexidine in treatment of pregnancy gingivitis.

Plaque and Gingival index were used to assess the oral health status of the subjects. Previous clinical trials have used these indices as effective parameters to evaluate the efficacy of the active components in preparations used in treatment of gingivitis (26-28).

Chlorhexidine is the gold standard for the treatment of gingivitis (23). It is also widely recommended for the treatment of pregnancy gingivitis (12). Previous studies have reported that the gel form of Chlorhexidine is more effective than the mouthwash (18). Hence, the gel form was used in this study, as it had an added advantage of better comparability with the ayurvedic medicament, as both are applied in similar manner.

G32 is an ayurvedic formulation, known for many years, effective in treating gingivitis $(20,21)$ and pregnancy gingivitis (22). These studies showed that scaling, curetting or surgical procedures for treating gingivitis could be avoided by the local application of G32. Similar findings were reported in this study. A mere local application of G32 had brought about 53\% reduction in dental plaque and $58 \%$ reduction in gingival inflammation.

The mean Plaque and Gingival Index scores reduced significantly over the trial period for both the groups. The difference in reduction of these scores in both the groups was not statistically significant, indicating that G32 is as effective as Chlorhexidine in reducing the plaque and gingival scores in this sample of pregnant females.

The participants were enquired about any side effects of the drugs experienced during the duration of one month trial. Few of the participants had complaint of nausea during this trial. These females gave a history of severe nausea during their first trimester. While some of them continued using the drugs, two of them withdrew themselves from the study prematurely.

Chlorhexidine formulations contain about $11.6 \%$ alcohol, as a preservative and a semi-active ingredient. Excessive use of alcohol based products cause allergy, desiccation of oral mucosa, staining and change in taste perception. A significant risk of developing oro-pharyngeal cancer exists with the long term use of these products (29). The use of these products in pregnant females is a matter of great concern. G32 is a water based product, causing none or minimal side effects of this nature, even on a long term use. It has a pleasant taste. Hence, the compliance of the patients would be better. Moreover, it was observed that G32 was economical to be used for a long duration than Chlorhexidine gel or mouthwash.

The major limitations of this study were the short duration of follow-up and small sample. Also, the sample could be drawn only from second trimester pregnant females due to ethical constraints. Further, no differentiation could be made for cases of 'Gingivitis' or 'Pregnancy Gingivitis', either through history or histologically.

Nevertheless, this study has evidently shown the effectiveness of G32 in treatment of pregnancy gingivitis. Future studies are recommended to evaluate the reduction in amount of bleeding, reduction of attachment loss, effect on initial caries and the microbiological changes brought about by G32. Also the effects on pregnancy outcomes can be examined in longitudinal trials in pregnant females. Additional larger studies are needed to determine whether these findings are applicable to other populations, and to the treatment delivered at other stages of pregnancy.

During the course of this study, it was noticed that most of the pregnant females did not perceive their oral hygiene status as poor. Many were not aware of any disease in their oral cavity, while others were not sure if they needed to get 
some treatment for their ailments. Moreover, quite a few females with gingivitis refused to undergo any treatment for their gingival disease. It was evident that pregnant females were not aware of the importance and the harmlessness of the oral hygiene procedures during pregnancy. Hence, there is a need to propagate the fact that the chemical methods of oral hygiene, like G32 and also Chlorhexidine, are safe and harmless to be used even during pregnancy.

\section{Conclusion:}

This study concluded that G32 gum-paint is effective in reducing plaque and gingival inflammation in pregnant females. It can be an efficient, safe and cost-effective alternative to Chlorhexidine in the treatment of pregnancy gingivitis.

\section{Acknowledgment:}

The G32 was sponsored by the Alarsin Company, Mumbai. The funding sponsor had no involvement in the design, analysis, or writing process.

\section{References:}

1. Barak S, Oettinger O. Common oral manifestations during pregnancy. Obstet Gynecol Sury, 2003; 58(9); 624-628.

2. Giglio JA, Lanni SM. Oral health care for the pregnant patient. J Can Dent Assoc Feb 2009; 75(1); 43-48.

3. Pinard A. Gingivitis in pregnancy. Dent Regist 1877; 31: 258-259.

4. Eiselt P. Gingival hypertrophy during pregnancy. Med Jahrb Osterr Staates 1840; 21: 560 .

5. DeLiefde B. The dental care of pregnant women. NZ Dent J 1984; 80: 41-43.

6. Jensen J, Liljemark W, Bloomquist C. The effect of female sex hormones on subgingival plaque. $\mathrm{J}$ Periodontol 1981; 52: 599-602.
7. Löe H. Periodontal changes in pregnancy. J Periodontol 1965; 36: 209-217.

8. Tumini V, Di Placido G, D’Archivio D. Hyperplastic gingival lesions in pregnancy. I. Epidemiology, pathology and clinical aspects. Minerva Stomatol 1998; 47: 159-167.

9. Hugoson A. Gingivitis in pregnant women - A longitudinal clinical study. Odontol Revy 1971; 22: 65-84.

10. Offenbacher S, Katz V, Fertik G. Periodontal infection as a possible risk factor for preterm low birth weight. $\mathrm{J}$ Periodontol 1996; 67: 1103-1113.

11. Offenbacher S, Jared HL, O'Reilly PG. Potential pathogenic mechanisms of periodontitis associated pregnancy complications. Ann Periodontol 1998; 3: 233-250.

12. Silk H. Oral Health in Pregnancy. Am Fam Physician Apr, 2008; 77(8); 1139-1144.

13. http://multimedia.3m.com. Peridex ${ }^{\mathrm{TM}}$ (Chlorhexidine Gluconate $0.12 \%$ Oral Rinse)

14. Meadows M. Pregnancy and the drug dilemma. FDA Consumer 2001; 35 (3). Available: www.fda.gov/fdac/features/2001/301 preg.html.

15. Khalessi AM, Pack AR, Thomson WM. An in vivo study of the plaque control efficacy of Persica ${ }^{\mathrm{TM}}$ : a commercially available herbal mouthwash containing extracts of Salvadora persica. Int Dent J Oct 2004; 54(5); 279-283.

16. Bajaj N, Tandon S. The effect of Triphala and Chlorhexidine mouthwash on dental plaque, gingival inflammation, and microbial growth. Int J Ayurveda Res Jan-Mar, 2011; 2(1); 29-36

17. Dodwad V, Kukreja BJ. Propolis mouthwash: A new beginning. J Indian Soc Periodontol Apr, 2011; 15(2); 121-125. 
18. Pai MR, Acharya LD, Udupa N. The effect of two different dental gels and a mouthwash on plaque and gingival scores: a six-week clinical study. Int Dent J 2004; 54(4); 219-223.

19. Radhakrishnan AN. G32 (Alarsin) as gum massage in 700 cases of bleeding gums and loss of stippling. Bombay Hospital Journal April, 1979; 21(2); 19-23.

20. Yadav NS, Waheeda SQ. A double blind trial of G32 in control of gingival inflammation and bleeding in 100 adult cases of Gingivitis and Periodontitis. J Ind Dent Assoc Jan 1981; 53: 13-15.

21. Wadhwa PK, Siddique MIA. G32 as local application in the treatment of gingivitis with bleeding gums and halitosis, after scaling and curetting. Maharashtra Medical Journal July, 1982; 29(4); 111-113.

22. Logeswari, Manjula, Kanthamani CR. Local use of G32 an ayurvedic drug in pregnancy gingivitis. The Antiseptic April, 1986; 83: 227-233.

23. Jones CG. Chlorhexidine: Is it still the gold standard? Periodontol 2000 1997; 15: 55-62.

24. Löe H, Silness J. Periodontal disease in pregnancy I - correlation between dental hygiene and periodontal condition. Acta Odontol Scand 1963; 21: 533-551.

25. Silness J, Löe H. Periodontal disease in pregnancy II - prevalence and severity. Acta Odontol Scand 1964; 22: 121-135.

26. Löe H, Schiott CR. The effect of mouthrinses and topical application of chlorhexidine on the development of dental plaque and gingivitis in man. $\mathrm{J}$ Periodontal Res 1970; 5: 79-83.

27. Jenkins S, Addy M, Newcombe R. Comparison of two commercially available chlorhexidine mouthrinses: II. Effects on plaque reformation, gingivitis, and tooth staining. Clin Prev Dent Nov-Dec, 1989; 11(6); 1216.

28. Brecx M, Netuschil L, Reichert B, Schreil G. Efficacy of Listerine, meridol and chlorhexidine mouthrinses on plaque, gingivitis and plaque bacteria vitality. J Clin Periodontol 1990; 17: 292-297.

29. McCullough MJ, Farah CS. The role of alcohol in oral carcinogenesis with particular reference to alcohol containing mouthwashes. Aust Dent J 2008; 53: 302-305. 